\title{
Are implementation interventions effective in promoting the adoption of evidence-based practices in stroke rehabilitation? A Cochrane Review summary with commentary
}

\author{
Aydan Oral \\ Department of Physical Medicine and Rehabilitation, Istanbul University, Istanbul Faculty of Medicine, Istanbul, \\ Turkey.E-mail: ayoral@istanbul.edu.tr
}

\begin{abstract}
.
BACKGROUND: Stroke is the leading cause of disability among neurological disorders. Evidence-based practices to reduce disability are presumed to be associated with more favorable outcomes.

OBJECTIVE: To assess whether implementation interventions are effective at increasing uptake of evidence-based practices in stroke rehabilitation.

METHODS: The Cochrane Review by Cahill et al. (2020) is summarized.

RESULTS: The effectiveness of implementation interventions on health professionals' use of evidence- based practices is uncertain.

CONCLUSIONS: There is an urgent need for future research investigating the utility of implementation interventions to ensure the uptake of evidence by health professionals for the benefit of stroke survivors.
\end{abstract}

Keywords: Evidence-based medicine, rehabilitation, stroke

The aim of this commentary is to discuss from a rehabilitation perspective the Cochrane Review "Implementation interventions to promote the uptake of evidence-based practices in stroke rehabilitation" by Cahill et al. (2020), ${ }^{\mathrm{a}}$ which was published by

${ }^{\mathrm{a}}$ This summary is based on a Cochrane Review previously published in the Cochrane Database of Systematic Reviews 2020, Issue 10, Art. No.: CD012575, DOI: 10.1002/14651858. CD012575.pub2 (see www.cochranelibrary.com for information). Cochrane Reviews are regularly updated as new evidence emerges
Cochrane Effective Practice and Organisation of Care Group. This Cochrane Corner is produced in agreement with NeuroRehabilitation by Cochrane Rehabilitation.

and in response to feedback, and Cochrane Database of Systematic Reviews should be consulted for the most recent version of the review.

The views expressed in the summary with commentary are those of the Cochrane Corner author(s) and do not represent the Cochrane Library or Wiley. 


\section{Background}

Stroke ranks first among neurological disorders in terms of disability-adjusted life years (GBD 2016 Neurology Collaborators, 2019), which means that stroke burden with relevance both to death and disability is high worldwide. Rehabilitation for stroke is well-known to reduce stroke-related disability regardless of age, gender, and stroke severity. Evidence is rapidly growing in stroke rehabilitation leading to a multitude of evidence-based guidelines (Platz, 2019), which might be helpful for optimal care of stroke survivors. However, rehabilitation professionals face challenges with implementing evidence into routine stroke care. Therefore, it is important to identify effective implementation interventions which enhance the uptake and use of evidence by rehabilitation professionals in their clinical practice. Implementation strategies may be diverse and may include educational materials, meetings, workshops, electronic resources, online modules, consultation with local opinion leaders such as mentors or experts, and reminders of evidence as well as audit, feedback and organizational initiatives for using evidencebased guidelines increasingly (Juckett, Wengerd, Faieta, \& Griffin, 2020). A Cochrane Review (Cahill et al., 2020) looked at evidence to see if implementation interventions were effective for increased use of evidence-based practices.

\section{Implementation interventions to promote the uptake of evidence-based practices in stroke rehabilitation (Cahill et al., 2020)}

\subsection{Objective}

The aim of this Cochrane Review was to evaluate the effect of interventions aimed at furthering the adoption of evidence-based practices (named as implementation interventions) in stroke rehabilitation.

\subsection{What was studied and methods}

The population addressed in this review was health professionals providing rehabilitation for survivors of stroke in a variety of settings (except for exclusively in acute stroke units) and the patients they cared for. The interventions studied were those intended to increase the use of evidence in stroke rehabilitation and those intended to produce behavior change in health professionals or stroke services or both with regard to evidence-based practices. The intervention was compared to no intervention or to another implementation intervention. The primary outcome studied was quality of care as measured using measures of adherence of the health professional to recommended evidence-based treatment. Secondary outcomes included patient, health professional, and resource use outcomes (e.g. patient's adherence to recommended treatment, his/her wellbeing and quality of life, health professional's intention for behavior change and professional satisfaction, cost-effectiveness, resources needed, and economic measures of long-term effects) along with adverse effects. The authors searched for both randomized and non-randomized trials, as they recognized that there are complexities associated with stroke rehabilitation and that there could be useful evidence within non-randomized controlled studies such as controlled before-after studies, in eleven databases including Cochrane Central Register of Controlled Trials, MEDLINE, Embase and others up to October 17, 2019.

\section{Results}

The review included 9 cluster randomized trials involving 12,428 patient participants, four of which were included in meta-analysis. No non-randomized trials met the inclusion criteria. The included trials were carried out in a wide variety of different settings and geographical locations (Australia, Canada, Malaysia, USA-1 trial in each of these countries and UK-5 trials) and explored the effect of a range of different implementation interventions for different members of the stroke rehabilitation team.

Within the context of stroke rehabilitation, the review shows that:

When comparing implementation interventions with no intervention

- There is uncertainty as to whether health professionals adhere to evidence-based practices better with implementation interventions, based on very low certainty evidence from two trials

- Patients' adherence to recommended treatment (based on low certainty evidence from one trial) and their psychological well-being (based on low-certainty evidence from two trials) may be no better with implementation interventions 
- Patients' function (activities of daily living) and health-related quality of life are probably no better with implementation interventions based on moderate-certainty evidence from two trials

- There were not any trials reporting how implementation interventions affected health professionals in terms of behavior change or satisfaction.

- Among five trials reporting economic outcomes, while one trial with high risk of bias reported that the implementation intervention was cost-effective, the other four trials did not.

- When implementation interventions were tailored to identified barriers, the results did not change.

When comparing an implementation intervention with another implementation intervention

- There is an evidence gap about whether one implementation intervention is more effective than another given there was limited relevant evidence and available data could not be combined due to heterogeneity.

\section{Conclusions}

The authors pointed to the uncertainty about whether implementation interventions improved adherence to evidence-based practice among health professionals providing rehabilitation for stroke survivors in comparison to no intervention or to another intervention.

\subsection{Implications for practice in neurorehabilitation}

Implementation science gains growing attention; yet implementation of evidence-based interventions in stroke rehabilitation is still lacking (Lynch, Chesworth, \& Connell, 2018). The results of the Cochrane Review by Cahill et al. (2020) reveal that it is still unclear whether implementation interventions improve the adoption of evidence-based practices by health professionals in stroke rehabilitation. Therefore, there is an urgent need for rehabilitation professionals to conduct robust research to elucidate the effectiveness of implementation interventions. However, in neurorehabilitation or in rehabilitation in general, other factors may also be relevant regarding implementation science. Recently, it is suggested that contextual factors such as health systems and service organization including health insurance availability, its coverage of interventions, as well as availability of technical equipment and qualified personnel for its use, may play a significant role in the implementation of evidence-based practice in rehabilitation in general (Gutenbrunner \& Nugraha, 2020). It may be suggested that future research need not only investigate the utility of implementation interventions such as educational interventions and/or audit or feedback further, but also those relevant to health systems and service organization. While there currently is a lack of robust evidence relating to the effect of implementation interventions, there remains a logical argument that strategies to enhance knowledge translation, through dissemination strategies such as the one Cochrane Rehabilitation has (Oral et al., 2020), might play an important role in enhancing uptake of evidence and delivery of optimal evidence-based rehabilitation to the benefit of stroke survivors. However, currently there is lack of evidence about the effectiveness of such knowledge translation strategies in supporting rehabilitation professionals to remain up-to-date with best evidence and their impact on quality of care. This area also poses a significant area of future research and it is challenging for organizations such as Cochrane Rehabilitation to develop and evaluate evidence-based strategies to enhance the uptake of evidence-based practices.

\section{Acknowledgments}

The author thanks Cochrane Rehabilitation and Mary Ann O'Brien and JC Han from Cochrane Effective Practice and Organisation of Care Group for reviewing the contents of the Cochrane Corner.

\section{Conflict of interest}

The author declares no conflicts of interest.

\section{References}

Cahill, L. S., Carey, L. M., Lannin, N. A., Turville, M., Neilson, C. L., Lynch, E. A., McKinstry, C. E., Han, J. X., \& O'Connor, D. (2020). Implementation interventions to promote the uptake of evidence-based practices in stroke rehabilitation. Cochrane Database of Systematic Reviews, 10, CD012575. doi: 10.1002/14651858.CD012575.pub2.

GBD 2016 Neurology Collaborators. (2019). Global, regional, and national burden of neurological disorders, 1990-2016: a 
systematic analysis for the Global Burden of Disease Study 2016. Lancet Neurology, 18(5), 459-480. doi: 10.1016/S14744422(18)30499-X.

Gutenbrunner, C. \& Nugraha, B. (2020). Decision-making in evidence-based practice in rehabilitation medicine: proposing a fourth factor. American Journal of Physical Medicine \& Rehabilitation, 99(5), 436-440. doi: 10.1097/PHM.00000 00000001394.

Juckett, L. A., Wengerd, L. R., Faieta, J., \& Griffin, C. E. (2020). Evidence-based practice implementation in stroke rehabilitation: a scoping review of barriers and facilitators. American Journal of Occupational Therapy, 74(1), 7401205050p17401205050p14. doi: 10.5014/ajot.2020.035485.
Lynch, E. A., Chesworth, B. M., Connell, L. A. (2018). Implementation-the missing link in the research translation pipeline: is it any wonder no one ever implements evidencebased practice? Neurorehabilitation and Neural Repair, 32(9), 751-761. doi: 10.1177/1545968318777844.

Oral, A., Arienti, C., Lazzarini, S. G., Grubisic, F., Kiekens, C., Negrini, S. (2020). The Cochrane Corners by Cochrane Rehabilitation. European Journal of Physical and Rehabilitation Medicine, 56(4), 529-534. doi: 10.23736/S19739087.20.06258-9.

Platz, T. (2019). Evidence-based guidelines and clinical pathways in stroke rehabilitation-An international perspective. Frontiers in Neurology, 10, 200. doi: 10.3389/fneur.2019.00200. 